\title{
Can Developmental AIS Provides Immunity to a Multi-cellular Robotics System?
}

\author{
Maizura Mokhtar and Yang Liu \\ Department of Electronics, \\ University of York, York YO10 5DD \\ $\{\mathrm{mm} 520, \mathrm{ylxx}\} @$ ohm.york.ac.uk
}

\begin{abstract}
The major challenge to multi-cellular robotics system is how to ensure the system is homeostatically stable. This position paper proposes a developmental artificial immune system (dev-AIS) framework that tries to provide and maintain homeostasis to the multi-cellular robotics system. If immunity is defined as the ability to maintain homeostasis; the dev-AIS framework will be designed based on the understanding and the abstraction of how different organisms attain for this property through evolution and developmental process. Early form of Innate Immunity evolve from the predator-and-anti prey relationship of the single-celled organism. Progress in evolution drove the evolution of $\mathrm{im}$ munity from this simple relationship to the development of the immune system in multi-cellular organisms.
\end{abstract}

\section{Introduction}

This position paper proposes the idea of a developmental AIS (dev-AIS) framework that may provide and maintain homeostasis of a multi-cellular robotics system autonomously. If homeostasis equates to immunity, the dev-AIS framework may describe how immunity of a complex multi-cellular system emerges from immunity of its smaller components; and how homeostasis of a complex system is modulated, whilst homeostasis of its smaller components remains unaffected. The dev-AIS provides for this ability by abstracting the evolution and developmental processes of immunity from the predator-and-anti prey relationship of single-celled organism to the multi-cellular and multi-organ biological immune system. The dev-AIS framework will be a framework that combines genetic regulatory network (GRN) with AIS.

\section{Objectives of the dev-AIS Framework}

If a robot is considered as a cell, and the multi-cellular robotic organism may resemble that of a biological multi-cellular organism. Immunity can be achieved (i) in singular robot: by applying predator-and-anti-prey relationship of single-cell organism, (ii) in swarm and aggregation mode: by applying specialisation of cells in early multi-cellular organisms such as Volvox and Dictyostelium discoideum 
and in (iii) aggregated mode: the regulation of functions for different immune cells in evolved multi-cellular organism may help regulate the functionality of each robot in the robotic organism. (i) and (ii) can be desribed using GRNs and (iii) uses AIS algorithms.

The predator and anti-prey relationship of a single celled organism (e.g. bacteria, ameoba) can describe the system management of a robot, responsible for efficient power and task distribution and homeostasis by predator: to solve a task within good time by making full use of available resources and anti-prey: to avoid running out of energy too quickly and/or creating deadlock, scheduling error or bottleneck to its processes.

The development and specialisation of cells in early multi-cellular organism can help describe the aggregation process; by defining how a shape and structure that is strong and stable is produced, whilst ensuring homeostasis for each robot remains unaffected (via GRN). Aggregation into a multi-cellular system is instigated, for example, when one robot cannot perform a task by itself because it has low energy, the task that is too demanding and requires additional processing capabilities and/or a robot is at fault. Such detections can be indicated by an AIS algorithm.

When the robots are aggregated in a single multicellular organism, it is more efficient that not all (individual) robots do similar action but instead each robot provides for its specialised functionality. How biological multi-cellular organism coordinate and modulate the interaction between the innate and adaptive immune system can be applied to the robotics system to help ensure good coordination of the specialised processes between each robots in the system. This includes anomaly detection and the definition of simple behaviour of individual robots (inherited) (Innate) and the response and adaptation to the environment (Adaptive).

\section{Summary}

If, homeostasis is the ability to maintain steady state operation despite any condition; in biology, homeostasis assimilates to immunity and is an emerging property. If we employ similar evolutionary and developmental processes of immunity in biology to a multi-cellular aggregated robotic system, similar properties may emerge. Homeostasis (immunity) of the aggregated robotic system may emerge because of the propagation of homeostasis of its individual robots. How these properties emerge and maintain in a multi-cellular robotic organism is through the developmental process described by genetic regulator network (GRN).

Evolution towards the best phenotype of the multi-cellular robotic organism is the product of the developmental process towards the best organism solution. This developmental process is the result of the integration of best single-celled robot's phenotype. The best phenotype of the single-celled robot has the GRN that describes for the best predator-and-anti-prey relationship of the robot with its environment. 